\title{
COMPACT High-POWER SHIPBORNE DOPPLER LidAR BASED ON High
}

SPECTRAL RESOLUTION TECHNIQUES

Songhua $\mathrm{Wu}^{1,2 *}$, Bingyi Liu ${ }^{1}$, Guangyao Dai ${ }^{1,3}$, Shenguang Qin ${ }^{4}$, Jintao Liu ${ }^{1}$, Kailin Zhang ${ }^{1}$, Changzhong Feng ${ }^{1}$, Xiaochun Zhai ${ }^{1}$, Xiaoquan Song ${ }^{1,2}$

${ }^{I}$ Ocean Remote Sensing Institute, Ocean University of China, Qingdao, China, *wush@ouc.edu.cn

${ }^{2}$ Laboratory for Regional Oceanography and Numerical Modeling, Qingdao National Laboratory for

Marine Science and Technology, China

${ }^{3}$ Leibniz Institute for Tropospheric Research, Liepzig, Germany

${ }^{4}$ Seaglet Environmental Technology, Qingdao, China

\begin{abstract}
The Compact High-Power Shipborne Doppler Wind Lidar (CHiPSDWiL) based on highspectral-resolution technique has been built up at the Ocean University of China for the measurement of the wind field and the properties of the aerosol and clouds in the troposphere. The design of the CHiPSDWiL including the transceiver, the injection seeding, the locking and the frequency measurement will be presented. Preliminary results measured by the CHiPSDWiL are provided.
\end{abstract}

\section{INTRODUCTION}

Atmospheric wind field has significant impact on atmospheric circulation, atmospheric carbon cycle, marine-atmosphere circulation and aerosol activities. Aerosols in the whole troposphere play a key role in climate change and air quality due to its direct, semi-direct and indirect effects on the radiation budget. It is the main driving force of various weather phenomena. To measure the characteristics of the optical properties, dynamics and the thermodynamics in the whole troposphere, the shipborne Doppler lidar based on High Spectral Resolution techniques is developed. Shipley [1] and Sroga [2] describe one of the first HSRLs. Eloranta [3] presents the principles of high spectral resolution lidar systems. Chanin et al. [4] presents the use of a dual Fabry-Perot interferometer (FPI) for Doppler frequency analysis. Korb et al. performed analyzes wind measurements with a single FPI [5] and a dual FPI [6-7]. Liu et al. [8] proposed the use of iodine vapor frequency discriminator for frequency analysis of backscattered light from both aerosol and air molecules for tropospheric wind measurements. Later on, a mobile ground-based incoherent Doppler wind lidar (MIDWiL) with an iodine filter was developed for the winds profiles from the signals of Mie and Rayleigh backscattering together in 2000. A van-based Doppler lidar using an injection-seeded diodepumped Nd:YAG pulsed laser with a high repetition rate was established to measure the sea surface wind with high spatial and temporal resolution for the Sailing Game. [9] The purpose of this paper is to introduce a Compact HighPower Shipborne Doppler Wind Lidar (CHiPSDWiL) based on High-SpectralResolution technique established at the Ocean University of China for the offshore measurement of the wind field and the properties of the aerosol and clouds in the troposphere.

\section{METHODOLOGY}

The atmospheric wind field can be retrieved from the combined Mie and Rayleigh backscattering by use of a direct-detect technology. The use of the second harmonic of Nd:YAG wavelength at 532 $\mathrm{nm}$, with adequate response to both Mie and Rayleigh scattering and a molecular filter as a frequency discriminator with $2 \mathrm{GHz}$ bandwidth. The wind measuring sensitivity is proportional to the slope of the filter transmission at an operating point. Another unique feature of the CHiPSDWiL system is the capability to monitor the aerosol scattering ratio, with which the radial wind can be calculated according to the aerosol load. With the aerosol-scattering ratio independently measured, the lidar returns passing through the frequency discriminator can be processed to retrieve Doppler wind profiles under a varied mixture of Rayleigh and Mie scattering. 
Figure 1 illustrates a schematic diagram of CHiPSDWiL, consisting of five major function blocks: telescope and scanner system, laser and seed injection system, frequency locking system, optical frequency discriminator system, and transceiver switch. All of the modules are integrated in a small container of $2 \mathrm{~m} \times 2 \mathrm{~m} \times 2.4 \mathrm{~m}$, excepting for the scanner, for the field experiment in a research vessel with limited spaces (Figure 2).
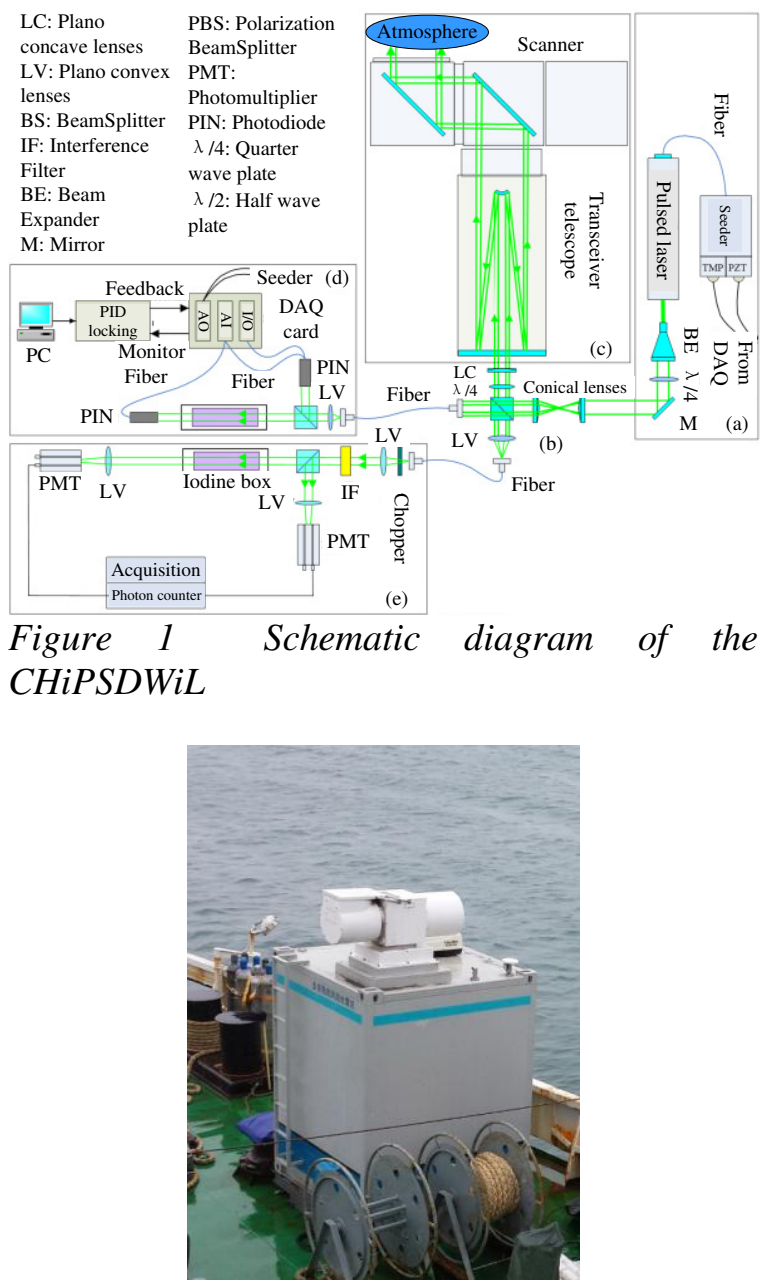

Figure 2 CHiPSDWiL installed on the research vessel Dongfanghong 2

The lidar transmitter provides high-peak-power and frequency-stabilized laser pulses at $532 \mathrm{~nm}$ by a diode-pumped, frequency-doubled, pulsed Nd:YAG laser, injection seeded by a seed laser and stabilized to an iodine absorption line. The laser repetition rate is $100 \mathrm{~Hz}$, and the single pulse energy is $\sim 125 \mathrm{~mJ}$ with pulse width of 10 ns. The seed laser is a continuous None-PlanerRing-Oscillator Nd:YAG laser and its $1064 \mathrm{~nm}$ output is injected into the pulsed Nd:YAG laser. The outgoing laser beam is firstly expanded to $250 \mu$ rad divergence by a $2 \mathrm{X}$ beam expander, and then directed into the transceiver telescope. A $300 \mathrm{~mm}$ diameter Cassegrain Dall Kirkham telescope with focal length of $3572 \mathrm{~mm}$ is the common part of the transmitting and the receiving optical path. Due to the structure blocking of the telescope, only the light beam in the annular space near the optical axis can be normally emitted, leading to much of the energy wasted. In order to solve this problem, for the first time to our knowledge, we use a pair of axicon lens together to produce an annular non diffractive Bessel beam to maximize the transmittance of laser beam and to minimize the unwanted back reflection and stray light. The annular beam transceiver simplifies the alignment between the transmitted beam and the receiver field of view and permits the lidar to operate with a $45-\mu$ rad FOV. Figure 3 shows the photos of transmitting annular beam at the surface of scanner and in the air.
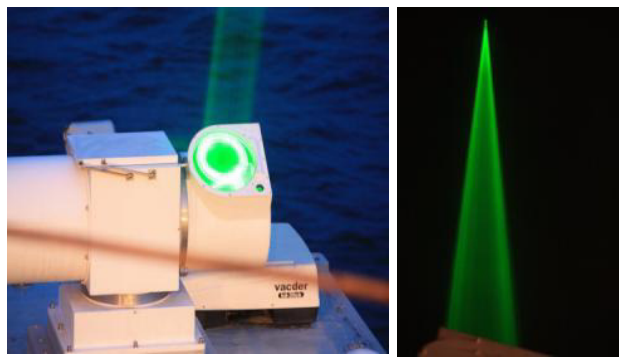

Figure 3 Lidar Scanner and the annular laser beam

A passive polarization transmit-receive switch consisting of a polarization beamsplitter and a $1 / 4$-wave plate is employed. The telescope directs the received photons through the quarterwave plate, which are converted into linear polarization with its axis of polarization perpendicular to that of the transmitted photons. So that the received photons pass through the polarizing cube. The received photons are then focused by lens onto the $200-\mu \mathrm{m}$-diameter multimode fiber that defines the receiver FOV. The fiber delivers the scattered light onto the high spectral resolution receiver system. After collimation by lens, scattered light passes through a skylight background filter comprised 
of a 0.1-nm interference filter followed by a non-polarizing beamsplitter. The signals are then split into two channels: one is detected directly by a photomultiplier tube (PMT) as a reference channel, and another goes through an iodine filter before being detected by another PMT in the measurement channel.

A small portion of leaked power from the transmitted laser beam through the polarizing beamsplitter is guided to a single mode fiber, which is directed to the second identical iodine cell and is locked to the $50 \%$ absorption level of the I2 line 1109 to stabilize the seed laser frequency. The frequency stability of the seed and amplified pulsed lasers are $200 \mathrm{kHz}$ and 1 $\mathrm{MHz}$, respectively. The spectral linewidth of the laser pulses is around $35 \mathrm{MHz}$.

The two-axis scanner provides coverage over an entire hemisphere $\left(0-360^{\circ}\right.$ azimuth and $0-180^{\circ}$ elevation). This scanner has the clear aperture of $320 \mathrm{~mm}$ and can accommodate co-axial transmission and reception with continuous scanning speed of $1-10 \%$ and directional accuracy of $0.1^{\circ}$. The scanning mirror has active stabilizing mechanism, which can compensate the pointing error caused by ship shake according to the information of orientation and attitude provided by the inertial measurement unit and GPS.

To measure the very large dynamic range of backscatter signal, an analog data acquisition and multi-channel scaler photon counting device is developed and used for the processes the data in real time with $10-\mathrm{m}$ resolution for the altitude range from $0 \mathrm{~m}$ to $20 \mathrm{~km}$ to retrieve wind products. It also controls the laser trigger, the optical chopper, the scanner.

\section{RESULTS}

After the CHiPSDWiL system was built up, we conducted atmospheric measurements for verifying its capability. Aiming at validating the wind direction and speed measured by the CHiPSDWiL, the concurrent observation by the CHiPSDWiL and radiosonde was operated. One of the comparison results is shown in Figure 4.

The measurement of wind profile compared with radiosonde on January 16, 2015 from 19:47 local time (LT) to 20:36 LT at the OUC Laoshan campus $\left(36.16^{\circ} \mathrm{N} 120.50^{\circ} \mathrm{E}\right)$. The Radiosonde data was from the Qingdao Observatory $\left(36.06^{\circ} \mathrm{N} 120.33^{\circ} \mathrm{E}\right.$, Station identifier of ZSQD and Station number of 54857) and measurement time began at 20:00 LT. The wind profile by lidar was compatible with the corresponding by radiosonde when the observation height was below $3 \mathrm{~km}$. Considering the range difference $(16.7 \mathrm{~km})$ between the CHiPSDWiL and radiosonde, the slight deviations of the wind direction and speed are acceptable.
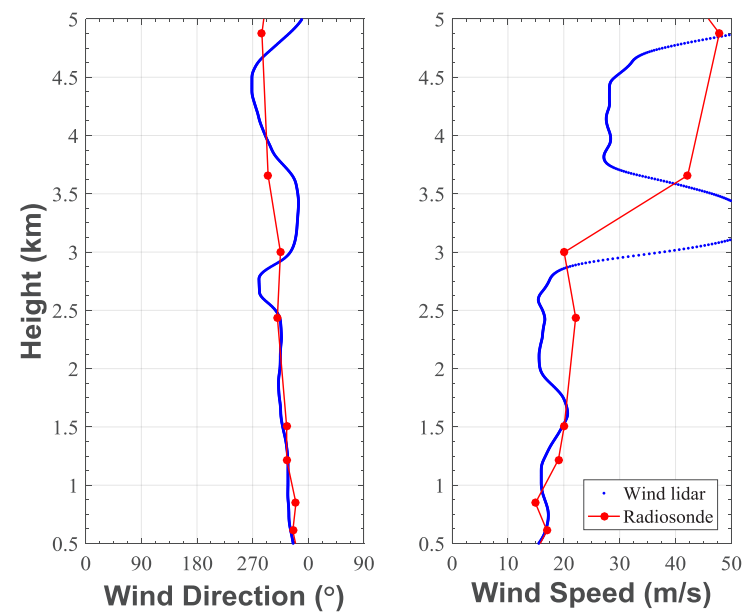

Figure 4 Comparison of profile on January 16, 2015. Lidar measurements were from 1947 local time (LT) to $2036 \mathrm{LT}$, and the radiosonde operated from $2000 L T$.

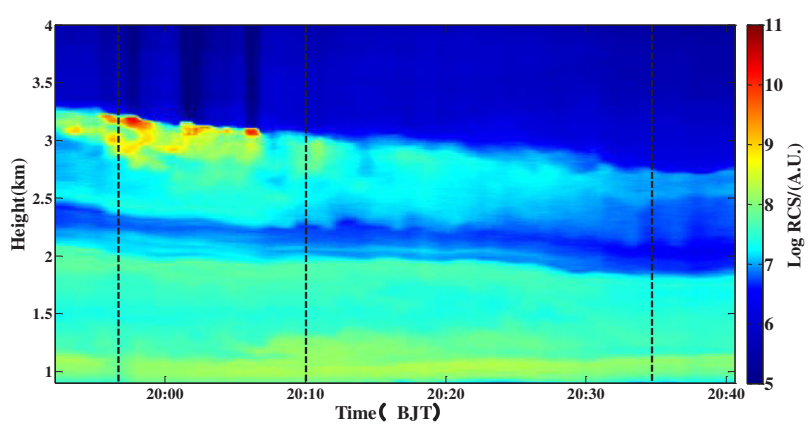

Figure 5 Range corrected atmospheric backscatter signal by CHiPSDWiL during 19:52 20:40 on 9 
April 2015
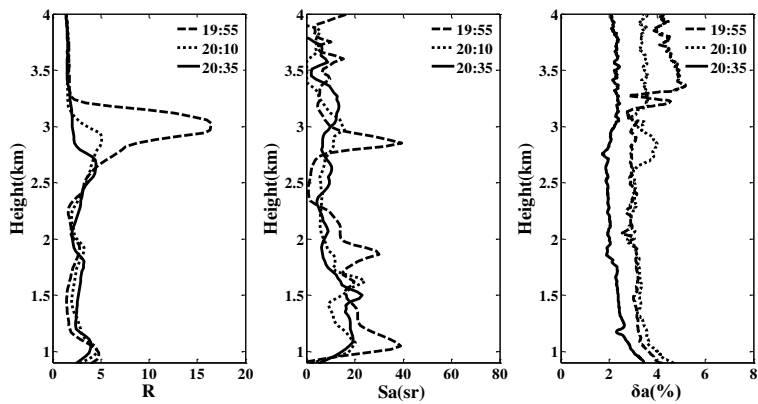

Figure 6 Profiles of aerosol backscattering ratio $(R)$, lidar ratio $(S a)$ and depolarization ratio $(\delta a)$ retrieved from five minutes' pulse accumulation signal at 19:55\20:10\20:35 on 9 April 2015

Figure 5 shows a range-squared backscatter measurement over the period 19:52 to 20:40 on April 9, 2015, presenting apparent aerosol layers and clouds. There are obvious clouds in the vicinity of $3 \mathrm{~km}$ and then gradually disappeared. In order to facilitate the analysis of the corresponding atmospheric conditions at different times, we take the characteristics of the experimental time at 19: 55, 20:10, 20:35 (Figure 5 black dotted line), respectively, with the accumulation time of $5 \mathrm{~min}$. The profiles of aerosol backscattering ratio (R), lidar ratio $(\mathrm{Sa})$ and depolarization ratio $(\delta a)$ are shown in Figure 6 . The value of $\mathrm{Sa}$ of the aerosol layer below 2 $\mathrm{km}$ is in the range of $10-25$, and $\delta \mathrm{a}$ is lower than $5 \%$, which is in accordance with the characteristics of marine aerosols. On the other hand, at an altitude of $3 \mathrm{~km}$, the value of $\mathrm{R}$ is 15 and $\delta \mathrm{a}$ is around $5 \%$ at the beginning of this observation, indicating a water cloud layer.

\section{SUMMARY}

We introduce a newly developed Doppler wind lidar/HSRL for measurement of wind profile and aerosol particle optical properties. The lidar transceiver transmits a unique Bessel laser beam into the atmosphere, offering a combination of advantages: (1) a high-peak-power seed injected DPSS laser and a normal aluminum coating DK telescope can be used to avoid laser damage and to improve the daytime observation capability. (2) Transceiver greatly releases the maintaining alignment between the laser beam and the receiver field of view and permits the lidar to operate in an unstable shipborne campaign.

\section{ACKNOWLEDGEMENTS}

This work was supported by the National Natural Science Foundation of China (NSFC) under grant 41375016.

\section{References}

[1] Shipley, S. T., Tracy, D. H., Eloranta, E.W., Trauger, J. T., Sroga, J. T., Roesler, F. L., and Weinman, J. A. 1983: High spectral resolution lidar to measure optical scattering properties of atmospheric aerosols. 1: Theory and instrumentation. Applied Optics, 22(23), 3716-3724.

[2] Sroga, J. T., Eloranta, E. W., Shipley, S. T., Roesler, F. L., and Tyron, P. J. 1983: High spectral resolution lidar to measure optical scattering properties of atmospheric aerosols. 2: Calibration and data analysis. Applied Optics, 22(23), 3725-373.

[3] Eloranta, E. W., 2005: Lidar: Range-Resolved Optical Remote Sensing of the Atmosphere, Claus Weitkamp ed., Springer Science+Business Media Inc, New York, USA, 143-164pp.

[4] Chanin, M. L., Gariner, A., Hauchecorne, A., Porteneuve, J., 1989: A Doppler lidar for measuring winds in the middle atmosphere, Geophys. Res. Lett. 16, 1273-1276.

[5] Korb, C. L., Gentry, B., Weng, C., 1992: The edge technique: theory and application to the lidar measurement of atmospheric winds, Appl. Opt. 31, 4202-4213.

[6] Korb, C. L., Gentry, B. M., Li, S. X., Flesia, C., 1998: Theory of the double-edge technique for Doppler lidar wind measurement, Appl. Opt. 37, 3097-3104.

[7] Flesia C., Korb, C. L., 1999: Theory of the double-edge molecular technique for Doppler lidar wind measurement, Appl. Opt. 38, 432440.

[8] Chen, W. B. Zhang, T. L., Hair, J. W., She, C. Y., 1997: An incoherent Doppler lidar for ground-based atmospheric wind profiling, Appl. Phys. B 64, 561-566.

[9] Liu, Z. S., Liu, B. Y., Wu S. H., Li Z. G., Wang Z. J., 2008: A high spatial and temporal resolution mobile incoherent Doppler lidar for sea surface wind measurements, Optics Letters 33(13), 1485-1487. 